\title{
Tendencias de cambio en la estructura social de América Latina y el Caribe hoy. Un debate interrumpido*
}

\author{
Giorgio Boccardo Bosoni**
}

\begin{abstract}
Resumen
El presente artículo tiene como objetivo establecer cuáles son las principales tendencias de cambio de la estructura social en América Latina y el Caribe para el periodo reciente. La transformación estructural ocurrida en esta región ha generado cambios significativos en la estructura social de estos países, y, a pesar de haberse recuperado una línea de investigación y reflexión respecto a la materia aún parece insuficiente. El problema estaría dado por la dificultad de pesquisar un tipo de modernización de carácter heterogéneo, que ha generado cambios en los agrupamientos sociales tradicionales, y que, a su vez, ha comenzado a constituir nuevos grupos sociales, de acuerdo a las modalidades que asume la transformación en cada uno de los países latinoamericanos.
\end{abstract}

Palabras clave: Estructura social - transformación estructural - tercerización - América Latina y el Caribe.

\begin{abstract}
The present article has been developed to show the mayor trends of structural change in Latin America and the Caribbean in the recent period. The structural transformation occurred in this region has generated significant changes in the social structure of these countries. Perhaps, it has been recovered a line of research and reflection on the subject, but it seems to be insufficient. The problem would be given by the difficulty of inquiring an heterogeneous type of modernization, that has generated changes in the traditional social groups, while these changes are becoming to constitute new social groups, according to every single characteristic that the process assumes in every Latin American country.
\end{abstract}

Keywords: Social structure - structural transformation - outsourcing - Latin America and the Caribbean.

* Agradezco las correcciones y comentarios del profesor Carlos Ruiz Encina que, en el marco de la guía de mi tesis de pregrado, animaron parte importante de este debate.

** Licenciado en Sociología de la Universidad de Chile. Investigador CIES. Docente del Programa de Bachillerato de la Universidad de Chile. 


\section{PROBLEMATIZACIÓN}

Desde su génesis, uno de los focos de interés fundamentales del conocimiento sociológico ha sido la relación existente entre un determinado modelo de desarrollo y los grupos sociales que lo sustentan. Dan cuenta de esto las diversas caracterizaciones y marcos analíticos desarrollados en torno a los procesos de racionalización y secularización ocurridos en las sociedades europea y norteamericana, y la determinación de los elementos necesarios para el establecimiento de un orden social, cualquiera fuera. A ello se suma la caracterización de los distintos grupos sociales y sus diversas orientaciones de acción relativas a dichos procesos.

Este debate ha sido alimentado por diferentes corrientes de pensamiento que han categorizado bajo distintas denominaciones a dichos grupos sociales. Se desarrollaron conceptos como los de clases, estamentos o grupos funcionales, y se definieron también las distintas relaciones de colaboración o antagonismo que estos grupos podían establecer entre sí. Sin embargo, a pesar de los enormes aportes entregados por estas escuelas de pensamiento ${ }^{1}$, su preocupación por establecer marcos explicativos de carácter general tuvo siempre limitantes históricosociales. Es así como la reflexión social latinoamericana del siglo XX se vio en la necesidad de desarrollar un pensamiento propio, que reconociendo la teoría social ya existente diera cuenta de la especificidad de América Latina y el Caribe, particularmente respecto al problema del desarrollo, o más bien a las condiciones estructurales que lo dificultaban.

Se apuntó, de forma crítica, a asumir los problemas y desafíos de la construcción de una sociología latinoamericana en relación con otras realidades continentales, dando lugar a escuelas de pensamiento que desarrollaron distintos marcos interpretativos, conocidos como la modernización, el desarrollismo o la dependencia. Dichos marcos contaron con una importante elaboración intelectual, tanto en la formación de principios teóricos como metodológicos, dando lugar a una mirada propia que, sin pretender negar la existencia de marcos interpretativos anteriores, se abocó a la conformación de un pensamiento social que buscara vincular la teoría con las sociedades latinoamericanas histórico-concretas.

Abundaron las investigaciones respecto al carácter de las oligarquías terratenientes, las burguesías nacionales, los grupos medios y el movimiento obrero, como también respecto al rol del campesinado y los grupos urbanos marginales en los procesos de modernización; a su vez, existió un interés por investigar las principales instituciones que aseguraban la reproducción o la generación de oportunidades de avance o retroceso para cada uno de estos grupos sociales (Solari et al., 1976). De esta forma, el problema del subdesarrollo estuvo ligado, en forma importante, al estudio de la estructura social de cada país, apuntando a determinar cuáles eran los grupos sociales que liderarían los procesos de modernización y cuáles serían también los grupos retardatarios de dichos procesos (Baño y Faletto, 1992).

En esa línea de reflexión destacaron autores como Gino Germani, José Medina Echavarría y Florestán Fernandes. Desde distintos paradigmas analíticos, estos autores desarrollaron una sociología vinculada a la investigación de las modalidades de desarrollo regionales y de los

\footnotetext{
1 Se hace referencia principalmente a las escuelas funcionalista, marxista y weberiana.
} 
grupos sociales que serían capaces de impulsar dichos procesos. Su preocupación se basó en la búsqueda de las condiciones para una dinámica de transformación, y en el análisis de los determinantes de la especificidad de las sociedades latinoamericanas en torno a dichos procesos (Fernandes, 1968; Germani, 1968; Medina, 1967, 1973).

Por su parte, autores como Faletto y Cardoso señalaron que el problema central era que en la región no se desarrollaba el capitalismo, sino un tipo específico de éste, lo cual los llevó a reconocer y caracterizar ciertos casos concretos y una tipología del capitalismo latinoamericano. Dicho análisis buscó dar cuenta de las características del desenvolvimiento de determinado modelo de desarrollo y los tipos de dominación asociados a éste, junto con establecer cómo las tensiones con los países capitalistas centrales, las grandes transformaciones del sistema capitalista global y las implicancias de la política internacional, afectaban la reorganización del orden político interno, el Estado y la reacción de los grupos sociales locales (Cardoso y Faletto, 1975). Sin embargo, bajo ningún punto de vista se pretendió hacer una lectura aislada de la región, ni tampoco asumir que la problemática latinoamericana no permitía una especificidad nacional. Más bien, se intentó responder a la pregunta por qué si la globalización, el desarrollo capitalista a nivel mundial y los ciclos económicos internacionales afectaban a todos los países del orbe, se daban en algunos casos nacionales situaciones tan disímiles, y en otros existían puntos de convergencia significativos.

Independiente de cuál fuera el enfoque teórico y metodológico para abordar el problema del desarrollo y la estructura social, un sello de esta sociología fue la construcción de una reflexión que superara las limitantes del enfoque económico como marco explicativo del problema del subdesarrollo, relevando la relación existente entre la acción de determinados grupos sociales y la estructura económica, tanto tradicional como en vías de modernización (Solari et al., 1976). No obstante, el desarrollo de la sociología latinoamericana sufriría una importante interrupción y transformación, a partir de la crisis del Estado de compromiso y la impronta autoritaria militar que afectó a la mayoría de los países de la región, entre las décadas de los setenta y ochenta. Tal situación provocó una disminución en el trabajo intelectual de algunos de los principales centros de pensamiento de la región (Faletto, 2003). A ello se sumó un cambio de orientación de la disciplina, desde una mirada más omnicomprensiva de la sociedad, a otra en donde predominaron los focos temáticos de investigación (Garretón, 1996). Así, la atención se centró en problemas tales como la transición a las nuevas democracias, las violaciones a los derechos humanos, la pobreza y la marginalidad, el género o la juventud; mientras otras temáticas como el desarrollo, la estructura social y los grupos sociales, y en general la preocupación por el modelo societal en su totalidad, pierden interés disciplinar.

Particularmente, adquieren un peso importante en los años ochenta los estudios sobre los grupos sociales más afectados por la crisis de la deuda, que se produjo en la mayoría de los países de la región. Fue así como abundaron las investigaciones sobre el crecimiento de la marginalidad urbana y la medición de la línea de la pobreza, cuyo enfoque será criticado a comienzos de este siglo, cuando se reabre el debate sobre estructura y estratificación social (Atria, 2001; Filgueira, 2004; Portes y Hoffman, 2003). Las críticas apuntaban tanto 
a que este tipo de estudios sólo observan a los grupos ubicados en el extremo inferior de la estratificación, no permitiendo visualizar la totalidad de la estructura social, como a que los indicadores para señalar la línea de la pobreza, a partir de una aproximación económica -como lo es el nivel de ingreso-, no abordan de forma cabal la complejidad de este fenómeno a nivel social, ni tampoco permiten aproximarlo al problema global del subdesarrollo (Filgueira, 2001).

De esta forma, la insuficiente explicación económica sobre las consecuencias que generó la desigual aplicación de las políticas de ajuste del denominado Consenso de Washington en la región reabrió el debate y la investigación respecto a los cambios en la estructura social de los países latinoamericanos, para el periodo reciente. Un esfuerzo importante lo realiza la Comisión Económica para América Latina y el Caribe (CEPAL) que desarrolló una línea de investigación en la materia, sistematizando tanto trabajos de índole teórica, como estudios empíricos de casos nacionales y regionales (Franco et al., 2008).

La gran mayoría de las investigaciones desarrolladas en la región en este periodo utilizan los esquemas interpretativos desarrollados por Erikson y Goldthorpe. Dicho esquema, de ocho categorías de clases sociales, se agrupa alrededor de las clases de servicio, intermedia y trabajadora (Erikson y Goldthorpe, 1992). También será utilizada la ampliación de la matriz clasificatoria original, incorporando criterios de propiedad y control de los medios productivos, prestación de servicios con mayor o menor autonomía, y diferencias de acuerdo a la manualidad del trabajo (Goldthorpe y MacKnight, 2003). Importante es señalar, además, que el esquema de clases propuesto por la denominada Escuela de Nuffield surgió producto del interés respecto a la discusión en torno a las consecuencias que tiene la movilidad social para la formación y la acción de clase en Inglaterra. Este esquema es esencialmente relacional, ya que enfatiza que las clases se definen en términos de los vínculos que establecen sus distintos componentes - de ahí la importancia de las relaciones de empleo-, a diferencia de los esquemas gradacionales que definen los grupos de acuerdo a una determinada cantidad de recursos: económicos, culturales o sociales. Para Goldthorpe, la movilidad social no conduce necesariamente a una sociedad más abierta, en que los individuos con diferentes orígenes tienen igual probabilidad de acceder a posiciones en las otras clases (Cf. Méndez y Gayo, 2005). Lo que se busca, más bien, es establecer cómo en la sociedad inglesa se constituyen grupos sociales en torno al proceso de modernización de la denominada economía de servicios, y como dichos grupos interactúan entre sí.

Ahora bien, esta preocupación no siempre estará presente en las adecuaciones del esquema propuesto por la Escuela de Nuffield, que en algunos casos se aplicará de forma mecánica para la medición de conglomerados sociales, sin necesariamente incorporar criterios de conformación histórica y grados de interacción entre los grupos sociales medidos. Es así como la heterogénea gama de estudios desarrollados en la región ${ }^{2}$ en la década actual, incluirá por ejemplo, una serie de estudios de casos nacionales gradacionales, entre los que destacan los

2 Para una revisión de los estudios citados ver Atria et al. (2007): Estratificación y movilidad social en América Latina. Transformaciones estructurales de un cuarto de siglo, LOM Ediciones, CEPAL, Santiago. 
realizados en Chile por Wormald y Torche, en Buenos Aires por Kessler y Espinoza, en Bolivia por Gray y Yáñez o en México por Cortez y Escobar. Otros, en cambio, han incorporado elementos de ajuste histórico y una orientación relacional, como los desarrollados en Chile por León y Martínez, o en Brasil por Do Valle Silva, sumados a los esfuerzos de Pérez y Andrade de medir la pobreza y el desempleo en Centroamérica. Finalmente, también existen aportes como el estudio de Mora y Araujo, que, a pesar de analizar particularmente el nivel social y económico, entrega un panorama nacional de la sociedad argentina.

Existe además un esfuerzo por recuperar una mirada regional de aproximación al estudio de clases sociales desde la perspectiva de ausencia o presencia de determinados activos sociales, evidenciado en análisis como los desarrollados por Portes y Hoffman. En dicha perspectiva se entremezclan los aportes realizados por Goldthorpe y los de Olin Wright, quien se centra en la noción de clase como dimensión de apropiación -explotación- y de control -dominación-, y donde cobran especial fuerza para el análisis de las clases las categorías de pequeño empresariado y trabajadores semiautónomos, en las cuales se cruzan relaciones capitalistas de producción típicas o genéricas con relaciones específicas de producción de pequeñas mercancías (Wright, 1989). Portes y Hoffman buscan diferenciar los estudios de estructura de clases realizados en sociedades desarrolladas, basados en criterios fundamentales como el control de los medios de producción, el trabajo de terceros y los recursos intelectuales escasos, de las posibilidades de investigación en América Latina, en donde una proporción importante de la población aún no ha sido incorporada a relaciones de trabajo legalmente reglamentadas e insertas de manera integral en el mercado de productos (Portes y Hoffman, 2003). Integran en su investigación tanto criterios de análisis de sociedades avanzadas como de condiciones estructurales propias del capitalismo periférico, que permiten un ordenamiento de los activos con que cuenta cada clase; así, se apuntó a establecer si los individuos tenían o no acceso a cada uno de estos activos -económicos, sociales, políticos y culturales- y si era posible la construcción de una tipología de la estructura básica de clase en América Latina.

A partir de lo señalado, se podría afirmar que se ha recuperado un interés disciplinar por el estudio de la estructura social a nivel regional, pero este interés parece aún insuficiente en el marco de la transformación que ha ocurrido en la región a nivel económico, político, social y cultural en las últimas décadas; particularmente desde finales de los ochenta en adelante (Baño y Faletto, 1999). Pero no basta con señalar en qué dirección se mueven los individuos de acuerdo a la tenencia o no de determinados bienes, ni tampoco señalar los cambios ocurridos en tal o cuál categoría ocupacional. El establecimiento de cuestiones tales como si los ascensos o descensos de dichos individuos se producen a partir de alguna característica común, o si en algunos espacios sociales se reproducen o son reclutados, o cuáles son los agrupamientos sociales que corresponden al modelo de desarrollo en curso y cuáles al modelo de desarrollo anterior, parecen como problemas importantes de recuperar en esta línea de investigación.

El problema estaría dado por la dificultad de pesquisar un tipo de modernización de carácter heterogéneo, que ha generado cambios en los agrupamientos sociales tradicionales, y que, 
a su vez, ha comenzado a constituir nuevos grupos sociales, de acuerdo a las modalidades que asume la transformación en los países latinoamericanos.

\section{LA TRANSFORMACIÓN ESTRUCTURAL. ALGUNOS ELEMENTOS DE CAMBIO A NIVEL REGIONAL}

A finales de los años sesenta, cuando concluye el desarrollo fácil de sustitución de importaciones, la expresión clásica del capitalismo limitado y dependiente se hace patente en la región, como también su incorporación parcial a las economías de los países centrales. De esta forma, el proceso de modernización resulta parcial y no se logra romper con el carácter dependiente latinoamericano de los centros económicos mundiales (Touraine, 1989). Posteriormente, a mediados de la década del setenta se produce a escala planetaria un reordenamiento del sistema capitalista, caracterizado por la emergencia de corporaciones multinacionales, sumado a un cambio significativo de la política internacional en los países de la región, cuya apertura comercial significó una conflictiva situación con los grupos medios y obreros que se habían desarrollado al alero del denominado Estado de compromiso (Faletto, 2003). A ello debe agregarse, la irrupción del campesinado y sectores urbanos marginales que presionaban al Estado por una mayor inclusión en las políticas sociales redistributivas (Cardoso y Faletto, 1975).

Es en dicho escenario de crisis regional en el que la mayoría de los países latinoamericanos inicia, bajo la impronta de los militares, una reorganización del orden económico, político y social; cambio que será impulsado por una nueva alianza social integrada por los militares y la tecnocracia estatal, la que dentro de los marcos del desarrollismo iniciará una apertura del modelo a capitales multinacionales como forma de reimpulsar las alicaídas economías nacionales (Atria y Ruiz, 2009). Dicha trasformación, que será impulsada desde el Estado en una modalidad conocida como burocrático-autoritaria, excluirá a importantes sectores medios y obreros, por la vía de la reducción del gasto público, la privatización de algunos servicios sociales y la reorganización de la relación entre el empresariado nacional y los trabajadores (O’Donnell, 1975).

Tal como se ha señalado, las décadas de los setenta y ochenta se caracterizaron por cambios en la modalidad del capitalismo dependiente latinoamericano, a excepción del caso chileno, que se mantuvo dentro de los marcos del desarrollismo. Se trató, sin embargo, de un desarrollismo menos nacional -sí estatal-, debido al ingreso de las multinacionales y la pérdida del peso de algunos sectores del empresariado nacional; como también menos popular, al excluir del modelo a importantes franjas medias y obreras que habían sido centrales en el periodo nacional-desarrollista. Pero la crisis no fue resuelta, traduciéndose más bien en un elemento que condicionará los procesos de redemocratización a finales de los ochenta. En particular, en cuanto dichos procesos de transición no significaron una vuelta a las antiguas democracias -nacional populares-, sino que se transformaron en "nuevas democracias" (Ruiz, 2006).

Es así como los países de la región inician el decenio de los noventa con el peso de la inercia recesiva de los ochenta, y con el pasivo que significó su deuda externa, condicionando la profundidad con la que se impulsó el paquete de reformas recomendadas 
por el denominado Consenso de Washington, como también los planes de "salvataje" de organismos internacionales como el Banco Mundial y el Fondo Monetario Internacional. Dicho paquete de reformas consistió en el restablecimiento de los equilibrios macroeconómicos nacionales, que incluyó pasar del modelo sustitutivo de importaciones a uno de apertura de mercados, la privatización de empresas del Estado, junto con ajustes al mercado laboral y a la cobertura de derechos sociales. Las transformaciones asumirán diversos grados de profundidad en los países latinoamericanos según las particularidades de sus situaciones nacionales, al punto que algunos casos se enmarcarán plenamente dentro del denominado giro neoliberal, mientras que en otros surgirá incluso un cuestionamiento acerca de si es la modalidad conocida como neoliberal la que caracteriza en forma más adecuada el panorama habido, o si se constituye en estos países una mezcla de las reformas de ajuste antes señaladas con rasgos propios del desarrollismo (Atria y Ruiz, 2009).

Se inicia un cambio en el modelo de desarrollo, en donde nuevamente se establece una primacía del modelo primario exportador, insertándose la región en el nuevo contexto de la economía global. De esta manera, habrá que resituar la problemática del desarrollo latinoamericano para poder establecer la pertinencia del análisis regional y la posibilidad de establecer ciertos ordenamientos nacionales y de bloques regionales, de acuerdo a las características que asume la modernización en cada situación particular, y las consecuencias que esto tiene en la respectiva estructura social.

\subsection{Algunos rasgos de la transformación económica}

Una tendencia que parece ser común a varios países latinoamericanos es que durante las últimas décadas el sector de la economía que más se desarrolla es el sector terciario o de servicios. Su crecimiento en la región no resulta menor, dado que durante los noventa las actividades del sector terciario aportaron nueve de cada diez nuevos puestos de trabajo, y a fines de esa década representaban un poco más de la mitad de la población económicamente activa (PEA) no agrícola, según la distribución del Producto Interno Bruto (PIB) (Weller, 2001).

A pesar de su gran peso en el mercado del trabajo y en la economía, el empleo en actividades terciarias presenta una enorme heterogeneidad -en composición y expansión- vinculada a cuestiones como las tendencias del desarrollo económico y productivo. Existe una serie de propuestas para la clasificación interna de dicho sector: por rama, por características del producto, o por propiedad de la empresa (Ibíd.). Pero, a pesar de tal heterogeneidad, parece ser consenso que se trata de actividades en las que el producto del trabajo presenta una difusa separación de al menos uno de los siguientes elementos: el proceso de trabajo, la distribución o el consumo final (Sayer y Walker, 1992). Esto hace que sean agrupables en una misma categoría actividades como la salud, la educación, el transporte, el comercio o los servicios inmobiliarios -donde el producto no se escinde del trabajo y el consumo-, con la industria energética y de tratamiento de aguas -donde la producción y distribución se intersectan- y la producción de bienes simbólicos -donde las fronteras entre producción, distribución y consumo se redefinen gracias al avance tecnológico. Así, su crecimiento y vinculación con 
múltiples áreas, tanto directa como indirectamente productivas, dificulta la asociación de este sector con la constitución de un grupo social homogéneo (Tokman, 2007).

Particularmente, Ilama la atención que el crecimiento del sector, comparado con la experiencia de los países avanzados, no parezca guardar relación con el grado de desarrollo de los sectores directamente productivos (Faletto y Baño, 1992). Lo relevante es que, a pesar de su crecimiento, se advierten ciertas tendencias de tercerización espuria (Pinto, 1984; Kessler y Espinoza, 2003), o generación de puestos de trabajo en servicios que requieren mano de obra no calificada, dando lugar a diversas modalidades de subempleo u ocupaciones informales, fenómeno que cobra mayor relevancia en las ciudades capitales, y que dista de forma significativa de los fenómenos advertidos en las sociedades desarrolladas y su tránsito a la denominada sociedad post-industrial (Bell, 1976). Esto remite nuevamente a la necesidad de caracterizar las modalidades que asume el capitalismo por estos lares y, a partir de ello, los rasgos de la transformación que la estructura social asume en los países de la región, que a su vez condicionan los respectivos estilos de desarrollo.

Por otra parte, comienzan a desarrollarse en este mismo periodo nuevos sectores industriales, en donde tienden a desaparecer las prioridades y las consideraciones de articulación interna de la estructura productiva, del mismo modo que criterios como la capacidad de difusión del progreso técnico o la autonomía dejan de ser elementos importantes en las elaboraciones de las políticas de industrialización, privilegiándose las actividades o rubros productivos capaces de encontrar algún lugar en el mercado internacional (Fajnzylber, 1983). Llama la atención la gran cantidad de empresas multinacionales que se instalan en los países de la región y que no son necesariamente de alta complejidad tecnológica, particularmente, por la poca capacidad de presión que tienen los grupos internos que han sido desplazados por la presencia de estos nuevos complejos industriales (Baño y Faletto, 1992).

Algunos sectores heredados de la etapa de la industrialización por sustitución de importaciones se transformaron de forma significativa en los años noventa, tras la apertura económica, y si bien su ponderación en las exportaciones totales es baja en casi todos los países de la región, la participación de las manufacturas en las exportaciones es significativamente mayor cuando éstas se dirigen hacia otros países latinoamericanos o caribeños, vinculadas a la industria manufacturera tradicional o de exportación, como ocurre en países de Centroamérica y México. Estas industrias operan bajo una gran variedad de regímenes -como la maquila, zonas francas o importaciones temporales para la exportación- modificando las formas de organización de la producción, y, por ende, de la estructura ocupacional y el régimen laboral en el sector industrial (CEPAL, 2008).

Surgen también ámbitos que alcanzan cierto grado de modernidad productiva vinculados a la exportación de bienes no tradicionales, formando islotes de modernidad que se caracterizan por establecer importantes niveles de desconcentración productiva, a través de modalidades de subcontratación y encadenamiento con otras empresas de bienes y servicios de menor tamaño (Atria y Ruiz, 2009). A pesar de que tienen fuerte inserción en determinados nichos económicos donde predominan como grandes productores, invierten en varios sectores, operan en varios mercados -externo e interno-, y permiten la articulación del capital financiero con 
el industrial. De esta forma, se reconfigura la distinción entre capital nacional y extranjero mediante un acelerado proceso de asociatividad asimétrica entre empresas multinacionales de países desarrollados y multinacionales de países en vías de desarrollo, con grupos de poder locales (Durand, 1997).

Respecto al sector pisci-silvoagropecuario, se constata una transformación significativa a modalidades industriales de desarrollo, dejando atrás las formas tradicionales de producción que caracterizaron a este sector durante gran parte del siglo XX latinoamericano. En los últimos decenios, la región ha estado marcada por una descomposición de la agricultura tradicional, y para muchos países de América Latina y el Caribe, el complejo agroalimentario resulta una fuente de productividad y de vinculación con otros sectores de la economía (CEPAL, 2008). Estos procesos de modernización del sector adquirieren importancia en cuanto a su participación en el PIB y las exportaciones, aunque no ha ocurrido lo mismo con la generación de ingresos y empleo (Favareto, 2009). Sin embargo, en algunos países la modalidad tradicional continúa imperando, y su población rural responde mayormente a los patrones tradicionales de exclusión y pobreza ${ }^{3}$ (Kay, 2009). Sumado a esto, en las últimas dos décadas se ha producido un crecimiento sostenido del sector forestal en la región ${ }^{4}$, resultado tanto de las políticas públicas como del incremento de la demanda de los países desarrollados (Alvarado, 2009).

A su vez, en el sector aparecen procesos de eslabonamiento hacia adelante, para la integración de nuevos productos y servicios relacionados con las cadenas de valor agroalimentarias, como también situaciones de eslabonamiento hacia atrás, en la generación de insumos para la agricultura -maquinaria, semillas, productos agroquímicos y servicios de asistencia técnica- a partir de la interacción con industrias tecnológicas de punta nacionales e internacionales (CEPAL, 2008).

En varios países de la región -como Bolivia, Brasil, Chile, México o Venezuela- los sectores mineros y/o energéticos han logrado generar cierto desarrollo tecnológico, que no se restringe a las grandes empresas de extracción de este tipo de recursos, extendiéndose a una amplia red de proveedores de bienes y servicios vinculados al sector. Asimismo, cabe destacar la actual tendencia a la internacionalización de algunas empresas de la región para asegurar mercados y participar en las actividades de transformación que generan mayor valor agregado, estableciendo canales de comercialización con los países desarrollados y algunas economías emergentes ( $I$ bíd).

\subsection{Algunos rasgos de la transformación del mercado laboral}

Más allá de las transformaciones ocurridas en las distintas ramas de la economía, las formas de empleo han cambiado de manera significativa en las últimas dos décadas a nivel general en la región. El mundo del trabajo aparece cada vez más heterogéneo internamente,

Es el caso de Bolivia y la gran mayoría de países centroamericanos.

Es el caso de Brasil, Uruguay, Argentina y Chile en el Cono Sur, y Costa Rica en Centroamérica. 
por efecto de procesos tales como la expansión de la precariedad salarial y la emergencia de nuevas modalidades de inserción laboral, la ruptura de las antiguas regulaciones que presidían las relaciones en el lugar del trabajo, o el retroceso de las actividades productivas industriales y el crecimiento de las actividades de servicios (Filgueira, 2001).

Tanto estas nuevas modalidades de inserción laboral como otras más antiguas, e incluso aquellas habitualmente ligadas a la economía de subsistencia, se integran a procesos ligados a los ámbitos más dinámicos de la actual modalidad de crecimiento, escenario que ya no resulta exclusivo de los trabajadores marginales, ni siquiera de los menos calificados, sino que se encuentra extendido en diversos estratos del mercado laboral (Ruiz, 2009). De este modo, los cambios obedecen tanto a un proceso de creciente segmentación al interior de la categoría genérica de asalariados, que transita desde un núcleo estable de trabajadores protegidos a una periferia -creciente- de trabajadores precarizados, como a la transformación de las formas de trabajo independiente, que resultan, en los hechos, cada vez más dependientes de la gran empresa moderna. Por otra parte, la flexibilidad aparece como otro rasgo distintivo del actual mercado laboral, afectando tanto las modalidades de dependencia económica, como la dimensión contractual del trabajo -dependencia e independencia-, y las modalidades de organización del trabajo según su carácter subordinado o autónomo (Filgueira, 2001).

A su vez, las formas de subcontratación se convirtieron, en los países de la región, en una de las modalidades más comunes de organización del trabajo, situación que genera una doble dependencia del trabajador, quien desarrolla su actividad bajo las normas de un establecimiento que no es parte de la empresa que lo contrata. Esta condición tiende a extenderse a múltiples sectores productivos, y se constituye como parte de procesos de encadenamiento creciente entre empresas, o como resultado de la tercerización de las actividades consideradas periféricas o auxiliares (Palominos, 1998). Se trata de una venta de servicios y no de un contrato laboral, o si se quiere, de una creciente mercantilización de las relaciones sociales.

Otro rasgo característico de la década de los noventa en América Latina y el Caribe es la creciente informalidad en la relación contractual. Dos de cada tres empleos creados en la región durante dicho periodo se generaron en el sector informal. Junto con ello, aumentaron los trabajadores sin contrato o con uno diferente al tradicional contrato por tiempo indefinido (Tokman, 2007). De esta forma, la temporalidad del empleo disminuye de forma importante, manifestándose en rasgos como la inestabilidad, la rotación y el alto nivel de creación y destrucción de puestos de trabajo en cortos periodos de tiempo, situación que da cuenta de los diferentes grados e intensidades con las que se expresa el fenómeno de la informalidad.

Lo relevante es que esta informalidad, más que aparecer como un hecho aislado, se expresa como una larga serie de encadenamientos entre el sector productivo formal y las distintas modalidades de subcontratación de bienes y servicios producidos en diversas modalidades contractuales, que van desde el empleo formal tradicional hasta el trabajo doméstico, como parte de una cadena descentralizada de grandes empresas formales, que en muchos casos son de carácter multinacional (Ibíd). Más que un fenómeno circunscrito a 
sectores específicos -agrícolas, industriales o de servicio-, lo que parece constituirse es una nueva base de organización del trabajo en la región, cuyos rasgos principales serían los grados de informalidad de la relación laboral, el encadenamiento y autonomía de las pequeñas y medianas unidades productivas de bienes y servicios vinculadas a la gran empresa, y la flexibilidad laboral, en sus dimensiones contractual, económica y organizacional.

Las transformaciones producidas en el mercado laboral, sumadas a la creciente tercerización -tanto espuria como genuina- de la economía de los países latinoamericanos, sugieren la necesidad de establecer importantes cambios en los criterios de análisis e investigación de la estructura social latinoamericana. Tales cambios deben considerar, entre otros elementos, el grado de profundidad con que la transformación estructural -en curso- es llevada adelante por algunos países, y la resistencia que presentan ciertos grupos sociales del periodo desarrollista, junto con los heterogéneos grados de constitución de los denominados grupos sociales que emergen de la transformación estructural.

\section{TENDENCIAS DE CAMBIO EN LOS GRUPOS SOCIALES DE AMÉRICA LATINA Y EL CARIBE}

Tal como se ha señalado, América Latina y el Caribe presentan una importante heterogeneidad respecto de las modalidades que asume la transformación reciente, tanto a nivel del sistema económico, como en lo referente al Estado y la estructura social. Luego, una caracterización de los grupos sociales debe dar cuenta de dicha transformación tanto en los grupos emergentes como en aquellos del periodo anterior, los que, dependiendo de cada país, presentarán diversos grados de desestructuración, reacomodo o resistencia. No obstante, dicha caracterización presenta algunas dificultades, debidas tanto a las matrices de análisis utilizadas, que no necesariamente han sido ajustadas a los cambios recientemente señalados, como a la heterogeneidad de los casos nacionales que dificulta la comparación y el agrupamiento a nivel regional. A ello debe agregarse la aún insuficiente cantidad y calidad de los estudios actuales, que en algunos casos se reducen a uno o dos por país, y que suelen abordar parcialmente la problemática de la estructura social.

Junto con lo anterior se debe considerar además que la existencia, y sobre todo la unidad de un grupo social determinado, es un supuesto que difícilmente se puede establecer a priori. Afirmar o negar esta condición implica analizar las diferentes unidades que lo compondrían, determinar su participación diferencial en la estructura de poder y/o prestigio y sus formas de reclutamiento y reproducción social; cuestión que no es posible resolver sólo con los actuales estudios -en su mayoría gradacionales-, que ubican a los individuos en casilleros a partir de la cantidad de bienes -económicos o culturales- que poseen, lo que impide atribuirles una determinada unidad, algún género de conciencia o un comportamiento unitario. Si bien los estudios de estructura ocupacional por lo general entregan algunos elementos de ajuste histórico y características relacionales, éstos resultan también insuficientes ante la magnitud de las transformaciones.

De esta forma, considerando la dificultad de delimitar con precisión -en este apartadouna caracterización global de los distintos grupos sociales, se apunta, al menos, a describir 
las principales tendencias de cambio de la estructura social de América Latina y el Caribe para el periodo reciente.

\subsection{Los sectores altos}

La mayoría de los estudios gradacionales y de estructura ocupacional consideran en la cúspide de la estructura social latinoamericana a los empresarios, bajo distintas denominaciones -empleadores, propietarios, capitalistas, etc. (Portes y Hoffman, 2005). Ahora bien, dicha categoría ha sufrido algunas modificaciones. Durante el periodo desarrollista, el empresario se asociaba principalmente a un propietario o alto gerente de una mediana o gran empresa ubicada en un sector moderno de la economía, que operaba con trabajadores asalariados libres, usando máquinas y equipos para producir bienes y servicios. Este concepto servía además, para distinguirlos de la vieja oligarquía o grandes propietarios que explotaban la renta de la tierra usando mano de obra campesina semilibre, con una base tecnológica heterogénea -en parte moderna y en parte tradicional.

En cambio ahora, iniciada la transformación estructural y superada la crisis de los noventa, se observa en la región una transformación en dicho sector. Los empresarios dependen menos del Estado y se han fortalecido como grupo económico y político, asumen funciones antes reservadas al sector público, comparten el predominio de la economía con el capital extranjero -producto de la privatización en curso-, cuentan con políticas laborales que fortalecen el margen de negociación de la gerencia, tienen una gama más amplia e influyente de instituciones empresariales, e incluso, en algunos casos, sus líderes se han convertido en ministros, asesores y hasta presidentes (Durand, 1997).

Sumado a esto, dada la situación dependiente de la región, en la mayoría de los casos la orientación del empresariado latinoamericano no estaría dada por la inversión en nuevas empresas, sino por el aprovechamiento de las facilidades que presenta el mercado, generando una actitud en ocasiones poco innovadora, lo que lleva a que en muchos casos predomine en ellos un comportamiento más propio del espíritu comercial y financiero. Buena parte de las investigaciones al respecto muestran que el comportamiento empresarial se caracteriza por una tendencia más adaptativa que por una opción transformadora (Baño y Faletto, 1992).

A continuación se ubican los altos ejecutivos, que cumplirían la función de administración del nivel superior en la mediana y gran empresa, tanto en el sector privado como en el público. Aunque no son propietarios directos del capital, manejan grandes organizaciones y controlan una voluminosa fuerza de trabajo organizada burocráticamente (Portes y Hoffman, 2005).

El siguiente escalón está integrado por algunas franjas de profesionales, definidos como trabajadores especializados con formación universitaria, empleados por empresas privadas e instituciones públicas en posiciones jerárquicas de alta responsabilidad. Si bien no controlan gran cantidad de capital ni grandes contingentes de trabajadores, su situación privilegiada deriva de la posición de conocimiento técnico escaso, requerido por las organizaciones privadas y públicas ( $\mathrm{Ibíd}$ ). Su proceso de conformación estaría dado a partir de los procesos de modernización de finales de la década de los setenta, y se les denominará como tecnocracia, 
por su orientación racional y de organización técnicamente eficaz de las instituciones (O’Donnell, 1981).

Este último grupo señalado se consolida en virtud de la gran crisis económica de los años 80 y sus tentativas de resolución que contribuyeron a no alterar la naturaleza tecnocrática del proceso de decisión en el modelo de desarrollo, el que se constituye como un burocratismo autoritario. Las nuevas democracias, en su mayoría, centralizaron la gestión de la economía en manos de un pequeño número de especialistas que formularon e intentaron implementar las políticas económicas sin consulta al Congreso, a los partidos y a los grupos organizados en la sociedad. Este sistema de equipos, actuando de forma aislada, buscó implementar políticas de ajuste procurando evitar las presiones de la sociedad y del resto del sistema político a través de una variedad de mecanismos. En el caso mexicano, por ejemplo, se puso en práctica por medio de una estructura burocrática fuerte, mientras que en otros países latinoamericanos se institucionalizó a través del apoyo personal de los presidentes o de un ministro fuerte (Loureiro, 1997).

Se señala, además, la significativa transformación del sector agrícola tradicional en algunos países (Favareto, 2009). Con ello se produce un cambio importante en uno de los grupos sociales que durante gran parte del siglo XX dominó de forma hegemónica la sociedad latinoamericana, a saber, la oligarquía (Bourricaud, 1969). La modernización del sector, sin dejar de resolver la situación de pobreza de millones de habitantes de las zonas rurales, llevó a que, en la mayoría de los casos, los propietarios de estos nuevos complejos agroindustriales adscribieran a patrones de comportamiento asociados a los grupos empresariales urbanos del sector moderno de la economía (CEPAL, 1992).

\subsection{Los sectores medios}

Otro sector de interés para la sociología latinoamericana está constituido por los grupos medios, fundamentalmente dado su rol tanto en el proceso político como en el proceso de modernización de la región. Dada la heterogeneidad y amplitud del concepto, las investigaciones han puesto el énfasis en la composición por estratos que registraron las distintas ramas de actividad, lo que vincula la expansión de este sector en América Latina al grado de desarrollo de las fuerzas productivas (Baño y Faletto, 1992).

Para el periodo desarrollista se distinguen al menos dos etapas de constitución de los sectores medios: el momento de su ascenso al poder, y el momento del compromiso, ligado a la mantención de la posición ya lograda. Además, se analiza particularmente tanto el origen de estas capas medias como sus relaciones con otros sectores sociales, señalándose la existencia de un grupo medio que proviene del periodo colonial, con un alto grado de dependencia respecto de los sectores altos, y otro que se formó en los albores del siglo XX, a partir del proceso de expansión de los sectores modernos de la economía. Los grupos medios emergentes se vincularon con los sectores populares, dado que la expansión de los primeros otorgaba también empleo a los segundos, y porque podían entregar un sustento ideológico a las demandas de los sectores populares (Graciarena y Franco, 1967). Los sectores medios, 
una vez consolidada su posición de privilegio en el Estado a partir de posiciones dentro de su burocracia, asumen una posición de mantención de las ventajas en empleos y acceso a bienes sociales básicos, y con ello la defensa del modelo de desarrollo de industrialización sustitutiva de importaciones (Baño y Faletto, 1992).

De esta forma, el énfasis de las investigaciones estuvo puesto en relacionar las alteraciones en tamaño, composición y perfiles de los sectores medios, con determinados patrones de movilidad social ocurridos en la estructura social de los países latinoamericanos. Los cambios en la proporción de los sectores medios sobre el total de la población económicamente activa se constituyeron en un importante indicador de modernización; de ahí la importancia que adquiere en las investigaciones de estructura y estratificación la categoría ocupacional (Filgueira y Geneletti, 1981).

Investigaciones más recientes, enfocadas en los sectores medios, han apuntando a una discusión más bien de tipo nacional y centrada en los rasgos de estratificación y movilidad, otorgándole importancia a elementos como el acceso a la educación y las pautas de consumo en la configuración de las trayectorias ocupacionales. A diferencia de los estudios de Filgueira y Geneletti en la década de los ochenta, estas investigaciones pondrán el foco de atención mayoritariamente en la movilidad individual, más que en la de tipo estructural. De todas formas, parece pertinente la revisión de dichos estudios nacionales, dado que permiten comparar si algunas de las transformaciones señaladas a nivel regional han generado tendencias de cambio en determinados grupos sociales, en direcciones comunes o disímiles.

Investigaciones realizadas en Argentina combinan la posición de las personas en las dimensiones educacional, ocupacional y de posesiones materiales en el hogar; obteniéndose resultados que señalan que se ha producido un cambio importante en la sociedad argentina durante los últimos años, desde una gran homogeneidad de su clase media y la existencia de una franja relativamente pequeña de personas en situación de pobreza, a una creciente segmentación al interior de los sectores medios y a un aumento importante de la pobreza (Mora y Araujo, 2001). Dicho tránsito ha generado una movilidad estructural ascendente, vinculada al aumento del peso de los puestos técnicos y profesionales de grupos medios heterogéneos, así como una reducción del empleo público y su recambio por servicios informales e inestables (Kessler y Espinoza, 2003).

La orientación asumida por la investigación anteriormente señalada no pareciera reflejar un caso particular de Argentina, pues investigaciones realizadas en Brasil también muestran la importancia que se le asigna a la educación en la posición social y en la determinación de los ingresos que obtienen los grupos sociales, particularmente los sectores medios. En este panorama, el nivel educacional aparece como un elemento central que define la situación de mercado de los individuos, y revela la fuerza que adquieren los títulos académicos (calificaciones, credenciales) en las posiciones ocupacionales y la determinación de ingresos en la sociedad brasileña (Do Valle, 2004).

En este mismo sentido, estudios realizados en Chile señalan que el distanciamiento interclasista en las participaciones en el ingreso total ha sido muy débilmente compensado 
por un acortamiento en las distancias educativas. Pese al aumento del promedio de los niveles educativos, comparada con la generación de sus padres, la actual generación de obreros presenta una distancia educativa levemente menor con los grupos medios; mientras los grupos marginales mantienen una distancia considerable respecto de los grupos medios y los grupos obreros. Al interior de la nueva generación, las distancias educativas interclasistas continúan siendo muy pronunciadas y permanecen como barreras difícilmente superables para la movilidad social (León y Martínez, 2004). El nivel educativo de los padres, aun más que los niveles de ingreso familiar, aparece como el principal determinante del nivel educativo alcanzado por los hijos, lo que indica la persistencia de fuentes principalmente adscriptivas de desigualdad.

Adicionalmente, este crecimiento significativo que se observa en los sectores medios se asocia principalmente a la expansión de la rama de servicios. Si bien no hay consenso en la literatura respecto a esta categoría señalada por Goldthorpe, ni menos respecto de si es posible una adecuación para países subdesarrollados como los latinoamericanos, es importante reseñar que en la década de los noventa la expansión del empleo en el sector terciario latinoamericano se basó en procesos simultáneos de inclusión y exclusión laboral, y una causa para ello podría ser la elevada heterogeneidad del empleo en este sector (Baño y Faletto, 1992): por un lado, el papel creciente de algunas actividades del sector terciario se expresó en la generación de empleos de productividad y calidad comparativamente elevadas; por otro, la expansión se produjo por la presión de la oferta laboral, y generó empleos que suelen ser poco productivos y de mala calidad (Weller, 2001). Incluso, en algunos casos, la expansión de los sectores medios estuvo vinculada al grado de participación de la mujer en el empleo, puesto que el sector servicios en general es responsable del grueso de la feminización del trabajo en el último tiempo (Lazzarto, 2001).

Este reciente debate ha sugerido que una parte de la expansión del sector terciario -aunque no su totalidad- responde al aumento de la economía informal, o de servicios de baja productividad y baja calificación (Weller, 2001; Gatica, 1986), cuestión que daría lugar a procesos de configuración de grupos medios en torno a una tercerización genuina; mientras que, asociado al proceso de tercerización espuria, se configuraría un grupo cuyo establecimiento global tanto en la categoría de sector medio como en la categoría de sector popular sería discutible.

Las principales aristas de esta discusión serían, en primer lugar, el problema de la productividad o dónde se ubican los servicios más productivos, y si, como conjunto, hay una productividad superior o inferior a la productividad media (Weller, 2004). Segundo, el problema de la calificación del empleo, o dónde y en qué intensidad la tercerización implicaría un aumento de la calificación del trabajo. Tercero, el problema de la equidad, que algunos autores sugieren respecto al fenómeno de la precarización laboral con un alto grado de informalidad, no producto de la exclusión, sino de encadenamientos a la economía de servicios, asociados incluso al trabajo calificado (Goldfarb, 2007; Baño y Faletto, 1999). Cuarto, el problema del género, por la feminización de la fuerza de trabajo de servicio en relación a otras ramas (Gálvez, 2004); y, finalmente, el problema de movilidad social, en 
términos de los alcances de los procesos de movilidad que abrió la expansión terciaria (Kessler y Espinoza, 2007).

Finalmente se hace referencia al surgimiento de un grupo de pequeños y medianos empresarios, tanto productivos como de servicios. Si bien existe aún poca investigación a nivel regional, en la mayoría de los países latinoamericanos se registró un apreciable incremento de los trabajadores por cuenta propia en el decenio de 1990 (Portes y Hoffman, 2005). Esta tendencia refleja el hecho de que antiguos asalariados del sector público se han visto "forzados" a dedicarse a actividades por cuenta propia, al declinar el empleo en esta área, y al no ser capaces de insertarse como asalariados en el sector privado.

En suma, los sectores medios, a pesar de su enorme tamaño en la estructura social en América Latina y el Caribe, han sufrido un cambio significativo en relación a la denominada clase media desarrollista. Además de haber dejado de participar en forma significativa del empleo público, aparecen como elementos de diferenciación interna su participación en el sector formal o informal, su condición de asalariados o trabajadores por cuenta propia, y su creciente participación en el sector terciario de la economía. Estas distinciones darían lugar a distintas fracciones medias cuyos espacios de configuración social serían completamente distintos, por lo que el ethos homogéneo presentado en el periodo desarrollista no sería reemplazado aún por uno nuevo.

\subsection{Los sectores populares}

Dada la denominación señalada anteriormente, correspondería a esta sección dar cuenta de los sectores bajos; sin embargo, la tradición sociológica latinoamericana ha prescindido en la mayoría de los casos de esta terminología, inclinándose más bien a hablar de sectores populares (Solari et al., 1976). Dichos sectores van desde el obrero tradicional hasta sectores campesinos y marginales, otorgándoles en muchos casos cierta categoría de agentes de cambio en el proceso de modernización latinoamericana. Cabe señalar también que en el periodo reciente ha adquirido relevancia el estudio de los sectores informales, cuyo tamaño en la población económicamente activa adquiere cada vez mayor importancia en los países de la región.

Diversos estudios respecto a los sectores obreros del periodo desarrollista destacaron las diferencias entre el denominado primer movimiento obrero, integrado mayoritariamente por inmigrantes europeos y con importantes niveles de autonomía respecto de la acción estatal; y el segundo movimiento obrero, integrado por inmigrantes internos no calificados, cuya presencia mayoritaria estaba ligada directamente a los regímenes populistas de gobierno que predominaron en algunos países de la región, y a las nuevas formas de industrialización que se asumen a mediados del siglo XX. Se establece además un alto grado de heterogeneidad interna de este grupo social (Germani, 1962).

Respecto a los datos que se manejan -entre la década del sesenta y el setenta-, se señala que el peso de este sector aumentó en la mayoría de los países; no obstante, en términos comparativos con el resto de la población económicamente activa, no siempre resultó 
decisivo en la estructura social y varió bastante en términos del país que se considerara. Aún así, pensando en el conjunto de los países latinoamericanos, el hecho es que después de la Segunda Guerra Mundial la migración interna pasó a ser un fenómeno masivo, y los nuevos contingentes que se formaron lo hicieron en el momento de expansión del consumo de masas, lo que influyó en la conformación de su tipo de demandas (Baño y Faletto, 1992). Se distinguen, para el periodo, dos estratos dentro de los sectores obreros: el alto, que estaba formado por individuos más calificados y con mayores posibilidades de movilidad social, por tanto más cercanos a los sectores medios; y el bajo, constituido por individuos con bajo nivel de calificación, y tendencia a las personalidades autoritarias y a participar de fenómenos de masas (Solari et al., 1976).

Estudios para el periodo reciente, y que aluden principalmente a casos nacionales, han señalado cambios en el componente y peso relativo de los sectores obreros en la estructura social de los países de la región. En el ya mencionado estudio de León y Martínez para el caso chileno, se señala la pérdida de peso estratégico de la clase obrera, y particularmente de la clase obrera industrial, en la estructura ocupacional, sumada a un cambio en su composición interna, disminuyendo las posiciones obreras en la industria y la construcción, y aumentando el "obrero" -manual y asalariado- en el comercio y los servicios (León y Martínez, 2004).

Do Valle, por su parte, al analizar los cambios en la estructura ocupacional del Brasil, señala que, al contrario de lo que ocurrió en el auge de los años sesenta y setenta, los sectores industriales dejaron de ser el motor de los cambios. De hecho, al examinar los dos estratos de trabajadores en la industria de manera agregada, comprueba que su proporción relativa disminuye ligeramente para las décadas del ochenta y el noventa, reducción que se observa tanto en las industrias "modernas" como en las "tradicionales" (Do Valle, 2004).

En otro estudio, Kessler y Espinoza, en un análisis de la sociedad argentina desde la década del sesenta hasta finales del siglo XX, evidencian también esta tendencia. Según los autores, el rasgo principal de la movilidad actual sería la influencia del alto peso de la clase obrera en las ocupaciones de origen. Aparentemente, la movilidad de circulación manifestaría reacomodos de los hijos de la clase obrera en la estructura social argentina (Kessler y Espinoza, 2007). Junto a esto, afirman que los hijos de obreros constituyen el grupo mayoritario en todas las categorías ocupacionales, excepto entre los empresarios; vale decir, todos los grupos ocupacionales tienen un significativo componente obrero. Se observa una disminución de los puestos obreros asalariados, y un recambio por puestos de servicios, especialmente en el comercio, así como en puestos profesionales, observándose en conjunto una pérdida de peso y un reacomodo de los obreros en la estructura social argentina (Ibíd).

La contracara de los sectores obreros la constituyó el campesinado latinoamericano, sector que adquiere relativa importancia de estudio a mediados del siglo XX, debido al atraso económico de la región y la presencia significativa del componente rural en la población económicamente activa. De esta forma, muchas investigaciones no necesariamente le atribuyeron al sector un ethos retardatario, sino que intentaron integrarlo al sector primario 
exportador en sus análisis (Solari et al., 1976). Los estudios de este tipo no sólo sirvieron para establecer diferenciaciones al interior del campesinado, sino que pretendieron constituir la base de explicación del surgimiento de movimientos campesinos, que se movilizaron en función del proceso de reforma agraria desarrollado en distintos países de la región.

Estudios más recientes señalan, por ejemplo, para el caso chileno, un cambio en el mundo rural. Más que el desaparecimiento del campesino, lo que se produjo fue una transformación importante en las condiciones del trabajador del sector agrario, quien pasó de distintos formatos del campesinado tradicional a trabajador asalariado, habitando en muchos casos en sectores urbanos y vinculándose a los complejos agroindustriales (PNUD, 2008). Por otro lado, el pequeño y mediano propietario rural se vinculó a dichos complejos, encadenándose de esa forma a los ciclos primario-exportadores que se desarrollan con fuerza en varios países de la región (Favareto, 2009). Por último, aún existen países donde el retraso y los patrones tradicionales de lo rural han pervivido, lo que mantiene a importantes franjas de la población en situación de pobreza y marginalidad (Kay, 2009).

Finalmente, el denominado sector informal ha sufrido importantes transformaciones para el periodo en curso. En el periodo desarrollista, la informalidad se vinculaba directamente con una condición de marginalidad, producto de la masiva migración campo-ciudad y la dificultad del sector moderno de la economía para absorber mano de obra a un ritmo adecuado, por lo que gran parte de los nuevos integrantes de la fuerza de trabajo, ya vinieran de procesos migratorios o del crecimiento vegetativo de la población, no encontraba trabajo en el sector moderno (Tokman, 1979).

En cambio, al finalizar la crisis de los 80, y producto de la aplicación de las reformas del denominado Consenso de Washington -con distintas variantes nacionales-, las nociones de informalidad, asociadas previamente a fenómenos como la pobreza y la marginalidad urbana, serán discutidas por organismos como la Organización Internacional del Trabajo (OIT) y la CEPAL, otorgándole al concepto una mayor complejidad, vinculada a la disminución de la regulación productiva y laboral que ocurrió en la década de los noventa en los países latinoamericanos (Tokman, 2007).

La situación de vulnerabilidad, antes asociada principalmente al sector informal, se hizo extensiva a gran parte de la PEA no agrícola, que trabaja en unidades productivas reconocidas dentro de los marcos legales y de regulación existentes, pero para los que las leyes no se aplican plenamente, sea por insuficiente fiscalización, legislación inadecuada o costos excesivos que obstaculizan contra su cumplimiento ( $/$ bíd). De esta forma se desarrolla una concepción más compleja de trabajador informal como un trabajador cuya relación de empleo, independiente de dónde trabaje, no está sujeta a los estándares establecidos por la legislación laboral, en aspectos tales como pago de impuestos, protección social y otros beneficios laborales. Esto significa, en definitiva, agregar al sector informal a todos los trabajadores no plenamente protegidos ocupados en empresas de más de 5 trabajadores (OIT, 2002).

Para el periodo comprendido entre 1990 y el 2005, el sector informal en América Latina y el Caribe crece hasta alcanzar más de la mitad de la PEA no agrícola. Sólo durante los tres 
años más recientes de dicho periodo se observa una estabilización en el tamaño del sector, asociado a la recuperación económica y del empleo con posterioridad a las crisis ocurridas a fines de los noventa y comienzos de la presente década ${ }^{5}$ (Tokman, 2007). Ahora bien, estas cifras regionales esconden una enorme heterogeneidad del sector informal, sugiriendo al menos la conformación de dos conglomerados de países. En el primer grupo, integrado por los países andinos y la mayoría de los centroamericanos, la informalidad alcanza entre la mitad y un poco más de dos tercios de la PEA no agrícola; mientras en el segundo grupo, integrado por los países del Cono Sur, además de México, Panamá y Costa Rica, ésta oscila entre un poco menos de la mitad y casi un tercio de la PEA no agrícola ${ }^{6}$ (Ibíd).

En este proceso se combinan problemáticas tradicionales -como la pobreza o la exclusióncon otras nuevas, como el encadenamiento de la informalidad o la precarización del trabajo calificado (Goldfarb, 2007), en donde ciertas fracciones sociales emergentes comenzarían a experimentar una serie de malestares similares a la crisis de la modernidad en el mundo desarrollado (Moulián, 1997), inaugurando una problemática social distinta a la tradicional pobreza o exclusión típica de algunos países de la región.

Estudios recientes establecen dos agrupamientos para el sector informal (Portes y Hoffman, 2003). El primero, a partir de la suma total de los trabajadores por cuenta propia, menos los profesionales y técnicos, más los trabajadores de las microempresas urbanas, de los pequeños establecimientos rurales, del empleo doméstico y de la mano de obra familiar no remunerada. El segundo ha sido ajustado tomando en cuenta que entre un tercio y la mitad de la población empleada en el sector formal presenta algún grado de informalidad. Resulta entonces que el segmento numéricamente más importante de la población empleada en América Latina y el Caribe es el que está excluido de relaciones laborales reglamentadas, seguridad social o salario fijo, a pesar de que se encuentra de una u otra forma encadenado a las relaciones capitalistas modernas.

Se expresa así la gran heterogeneidad de este sector, que incluye al trabajador asalariado sin vínculo legal, a los trabajadores autónomos y a los pequeños empresarios y propietarios, y que supone importantes diferencias en las formas de su inserción en el proceso productivo. Por ejemplo, en el caso de la economía brasileña, una de las causas del mantenimiento y la ampliación del sector informal sería la coexistencia de una oferta abundante de mano de obra con una detallada reglamentación y protección del trabajo asalariado, que llevaría a las empresas a recurrir a mecanismos para esquivar las restricciones a la flexibilidad y abaratar

5 Para el 2005, un 55\% de los trabajadores informales desempeñaba trabajos por cuenta propia, 33\% se encuentra en microempresas de menos de 5 trabajadores y el resto, $12 \%$, se ocupa en el servicio doméstico. Los ocupados por cuenta propia y las microempresas aumentan su participación, mientras que el servicio doméstico se mantiene estable. De los 2,8 puntos porcentuales de aumento de la informalidad durante los 15 años, 1,5 proviene de las microempresas y 1,2 del crecimiento de los trabajadores independientes.

6 En el primer grupo el sector informal oscila entre un máximo de $71 \%$ en Bolivia a un mínimo de $55 \%$ en El Salvador, con un promedio de $61 \%$. En un segundo grupo, en donde el sector informal registra niveles inferiores al promedio regional, se encuentra Chile con un 32\%, que es el mínimo para la región, hasta un rango de 43-44\% donde se ubican Brasil, México, Panamá y Uruguay; en Costa Rica el sector informal alcanza a 40\%, mientras que el promedio para el grupo en su conjunto alcanza a $41 \%$. 
la mano de obra (Do Valle, 2004). El principal mecanismo de articulación entre los sectores formal e informal es la subcontratación de servicios, indicando que la informalidad tiene un espacio propio en la producción, la que aumenta cuando la flexibilidad productiva y la reducción de costos de mano de obra se tornan más significativas ( $I b i ́ d)$.

Lo anterior evidencia nuevamente que las distinciones clásicas de la sociología desarrollista resultan poco esclarecedoras para este nuevo periodo. La tradicional dicotomía entre inclusión y exclusión, central en los albores de la sociología del desarrollo, poco puede decirnos acerca del encadenamiento que una fracción de la economía informal podría tener con el sector servicios (Katzman, 1984). Por ejemplo, parecería poco pertinente que trabajadores semiautónomos de una PYME de servicios vinculada con las grandes empresas formales, sólo por su condición de informalidad, se situaran en los sectores más bajos de la estructura social.

De esta forma, tampoco sería posible asumir que ciertas fracciones de asalariados - de baja calificación-, en general, debieran constituir un grupo social homogéneo dentro de los sectores populares, por el solo hecho de compartir una relación laboral similar o depender de su trabajo para la obtención de los ingresos. Las diferencias entre asalariados en el sector informal y en el formal son significativas, tanto por sus remuneraciones como por la protección, relación contractual o nivel de vinculación con el sector moderno de la economía.

\section{CONCLUSIONES}

Como se ha señalado en los apartados anteriores, la transformación estructural ocurrida en América Latina y el Caribe a mediados de la década de los ochenta e inicios de los noventa se caracterizó por la crisis de la deuda, y por la posterior aplicación heterogénea de reformas estructurales recomendadas por el ya mencionado Consenso de Washington; reformas que se implementan, en diferentes grados, en el contexto de la dilatada crisis del periodo desarrollista.

La década de los noventa se inició con un resistido proceso de cambio que impactará y modificará de forma significativa la estructura social latinoamericana. Grupos sociales del periodo desarrollista en proceso de reacomodo o desestructuración, como también nuevos conglomerados sociales, que comienzan a configurarse en el periodo reciente, van a constituir la estructura social en la región. Las transformaciones no serán homogéneas, y estarán relacionadas tanto con el grado de profundidad con que se implementaron las políticas de ajuste estructural como con el grado de resistencia de los grupos sociales del periodo desarrollista.

En esta dirección, las investigaciones sobre estructura y estratificación social realizadas a finales de la década de los noventa e inicios del presente siglo han permitido reinaugurar un debate interrumpido desde hace casi dos décadas. Sin embargo, falta profundizar sobre fenómenos emergentes que no parecen expresarse aún de forma tan nítida en dichos estudios, sin duda debido a que los tiempos del proceso histórico, aún en curso, demoran más en impactar la estructura social de los países que la estructura económica o política; como 
también, dado que los marcos analíticos y de medición en las investigaciones de estructura y estratificación social no parecen ser lo suficientemente sensibles a dichos cambios.

Aparecen de esta forma grandes conglomerados sociales que, si bien se vinculan a un determinado sector productivo o se encuentran bajo determinada condición laboral, esconden una enorme heterogeneidad de situaciones, que es necesario revisar y desagregar. También resulta necesario estudiar, en algunos países latinoamericanos, la significación de categorías sociales que antaño tenían un enorme peso en la estructura social, y que hoy, prácticamente desaparecidas, siguen registrándose como categorías fundamentales.

En buenas cuentas, se pretende establecer que la tradicional dicotomía campo y ciudad, o el paradigma de la modernización, dejan en cierta medida de constituir el marco explicativo fundamental de la sociedad latinoamericana. Con esto no se quiere señalar que la gran industria desarrollista o los grupos asociados a dicho paradigma -clase media asalariada estatal o clase obrera- hayan desaparecido, sino más bien que, en el marco de la transformación reciente, dejan de tener el peso estratégico que tenían antaño.

De ahí que se haga necesario revisar ciertos procesos de la transformación reciente, para poder recuperar críticamente este viejo problema sociológico entre el modelo de desarrollo en curso y los grupos sociales que lo encabezan o dificultan. Es en esta dirección que se puede observar el creciente peso que adquiere el sector terciario tanto en la producción como en la estructura ocupacional, no sólo en los servicios asociados a la producción industrial -como en la etapa desarrollista-, sino más bien en un nuevo tipo de servicio, tanto genuino como espurio, que comienza a constituir una suerte de "capitalismo de servicio", que impacta de forma significativa la estructura social en todos los niveles.

Por otro lado, resulta importante el cambio ocurrido en la base organizacional del mercado laboral. Se desarrollan, en distintos grados, cambios en la formalización de la relación laboral. Contratos más cortos, cambios en la duración de la jornada, pérdida de la seguridad laboral y social, salarios variables, distintos grados de flexibilidad y una difusa frontera entre los trabajadores autónomos y los dependientes, resultan expresivos de esta nueva forma de organización del trabajo.

La subcontratación es otra de las caras de este fenómeno. Se desarrolla de manera creciente un tipo de pequeña y mediana empresa (PYME), tanto de bienes como de servicios, que cumple, o funciones de producción que la gran empresa ha externalizado, o funciones de administración de trabajadores, que desarrollan sus tareas en un establecimiento cuyos propietarios no son sus empleadores. Así, cada vez más las fronteras entre el trabajo formal e informal, como también entre el sector moderno y el tradicional, se diluyen en encadenamientos que los vinculan. Se observan situaciones en las cuales la economía moderna demanda a las PYMEs o a los trabajadores domésticos bienes y servicios que antes producía internamente, alterando las viejas nociones de "integrados y excluidos" al modelo de desarrollo.

Ahora bien, todos estos cambios van modificando de forma importante la estructura social y la configuración de los grupos que la conforman. Se puede observar un cambio importante 
en el empresariado, tanto por su configuración más diversa, multisectorial y multinacional, como por el peso que adquiere tanto en el conjunto de la estructura social como dentro del denominado sector alto. La apertura económica de los países latinoamericanos, junto con el ingreso de las multinacionales, ha desdibujado las barreras del capital nacional y extranjero. De todas formas, se constituyen conglomerados nacionales -que en algunos casos cuentan con socios extranjeros- cuyo campo de acción se extiende desde el campo industrial al agrícola, financiero y/o de servicios.

Llama la atención la consolidación de la denominada tecnocracia como grupo social, que, sin tener el control de la propiedad ni administrar grupos de trabajadores organizados burocráticamente, logra cierto nivel de cohesión y un ethos vinculado a la idea de la planificación -principalmente de programas estatales- racional y técnicamente eficaz, por fuera de las presiones de distintos grupos sociales. Por su parte, los sectores medios dejan de ser un grupo homogéneo vinculado al crecimiento del empleo profesional en el sector público. Más bien, aparecen como un sector heterogéneo, tanto en los asalariados -principalmente del sector privado- como también en los trabajadores por cuenta propia, cuyo crecimiento principal está vinculado a la expansión del sector terciario de la economía.

Por último, se constata una pérdida de peso significativo del campesinado tradicional y la clase obrera desarrollista. En su gran mayoría, los primeros se transformarán en trabajadores asalariados de la agroindustria; mientras que los segundos en asalariados principalmente del comercio o de servicios -tanto formales como informales. Sumado a esto, la propia noción de marginalidad pierde peso dando paso a la de trabajador temporal, que, sin resolver en muchos casos su situación de pobreza, se integra bajo distintas modalidades al sector moderno de la economía.

En suma, a pesar de la enorme heterogeneidad que presenta la estructura social de los países latinoamericanos, aparecen ciertas tendencias comunes de cambio. Destacan dentro de éstas la tercerización -espuria y genuina-, los grados de informalidad de la relación contractual, la subcontratación -tanto de bienes y servicios, como de mano de obra-, la pérdida de peso de las capas medias y obreras desarrollistas, las transformaciones del mundo rural, y el surgimiento de un nuevo empresariado.

Esto permite pensar en la posibilidad de establecer nuevas investigaciones cuyos marcos analíticos y metodológicos expresen de forma más nítida tales cambios, permitiendo establecer con mayor precisión una caracterización de los grupos que componen la estructura social en la región; como también, poder esclarecer las modalidades que asume el capitalismo latinoamericano -si es que es posible hablar aún de esta unidad-, con sus respectivas variantes nacionales, y su vinculación con las transformaciones en curso a escala planetaria. Se apunta de esta forma a recuperar una sociología que, sin perder de vista la mirada histórica, pueda volver a mirar la sociedad desde una perspectiva de totalidad. 


\section{BIBLIOGRAFÍA}

Alvarado, Raquel (2009): “La expansión forestal en el Cono Sur. Políticas públicas, intereses transnacionales y transformaciones territoriales", Nueva Sociedad, № 223, septiembre-octubre, pp. 76-93.

Atria, Raúl (2004): "Estructura ocupacional, estructura social y clases sociales", CEPAL, División de Desarrollo Social, Serie Políticas Sociales, № 96, Santiago, octubre.

Atria, Raúl y Carlos Ruiz (2009): “Política y transformación social en América Latina: descentración de la acción estatal e ilusión tecnocrática", Ponencia presentada para el Congreso Mundial de Ciencia Política, Santiago.

Atria, Raúl, Rolando Franco y Arturo León (coord.) (2007): Estratificación y movilidad social en América Latina. Transformaciones estructurales de un cuarto de siglo, LOM Ediciones, CEPAL, Santiago.

Baño, Rodrigo y Enzo Faletto (1999): "Transformaciones sociales y económicas en América Latina", Universidad de Chile, Cuadernos del Departamento de Sociología, Santiago.

(1992): "Estructura social y estilo de desarrollo", Universidad de Chile, Departamento de Sociología, Serie Cuadernos de Trabajo, № 2, Santiago.

Bell, Daniel (1976): El advenimiento de la sociedad postindustrial, Alianza Universidad, Madrid.

Bourricaud, Favre (1969): Notas acerca de las oligarquías en Perú, Amorrortu, Buenos Aires.

CEPAL (2008): La transformación productiva 20 años después. Viejos problemas, nuevas oportunidades, CEPAL, Santiago.

Do Valle, Nelson (2004): "Cambios sociales y estratificación en el Brasil contemporáneo (1945-1999)", CEPAL, División de Desarrollo Social, Serie Políticas Sociales, № 89, Santiago, julio.

Durand, Francisco (1997): "Nuevos empresarios (y algunos viejos problemas)", Nueva Sociedad, № 151, septiembre-octubre, pp. 1-16.

Faletto, Enzo y Fernando Henrique Cardozo (1975): Post Scriptum a "Dependencia y desarrollo en América Latina, Centro de Estudios de Estado y Sociedad (CEDES), Buenos Aires.

Faletto, Enzo (2003): "La dependencia y lo nacional popular", Revista de Sociología, № 17, Facultad de Ciencias Sociales, Departamento de Sociología, Universidad de Chile, pp. 9-22.

Fanjzylber, Fernando (1983): La industrialización trunca en América Latina, Editorial Nueva Imagen, México.

Favareto, Arilson (2009): "La nueva ruralidad brasileña. Lo que cambió (y lo que no cambió) en el ámbito rural", Nueva Sociedad, № 151, septiembre-octubre, pp. 146-163.

Fernandes, Florestán (1968): Sociedades de clases y subdesenvolvimiento, Zohar Editores, Rio de Janeiro.

Filgueira, Carlos (2002): “La actualidad de viejas temáticas: sobre los estudios de clase, estratificación y movilidad social en América Latina", CEPAL, División de Desarrollo Social, Serie Políticas Sociales, №59, Santiago.

Filgueira, Carlos y Carlo Geneletti (1981): "Estratificación y movilidad ocupacional en América Latina", Cuadernos de la CEPAL, № 39, Santiago.

Gálvez, Telma (2004): “Relaciones laborales de Nuevo Tipo. Sistematización, detección y medición de problemáticas de género de nuevo tipo", SERNAM, Documento de Trabajo, № 86, Santiago. 
Garretón, Manuel Antonio (1996): “¿Crisis de la idea de sociedad? Las implicancias para la teoría sociológica", Revista de Sociología, № 1, Facultad de Ciencias Sociales, Departamento de Sociología, Universidad de Chile.

Gatica, Jaime (1986): "La evolución del empleo formal e informal en el sector servicios latinoamericano", PREALC, Documento de Trabajo, №279, Santiago.

Graciarena, Jorge y Rolando Franco (1981): Formaciones sociales y estructura de poder en América Latina, Paidós, Buenos Aires.

Germani, Gino (1968): Política y sociedad en una época de transición, Paidós, Buenos Aires.

Goldfarb, Enrique (2007): No todo está perdido, la encrucijada de las PYMES y de la clase media, Tajamar, Santiago.

Kay, Cristóbal (2009): "La persistencia de la pobreza rural en Honduras, Nicaragua y Bolivia: un fracaso del neoliberalismo", Nueva Sociedad, № 223, septiembre-octubre, pp. 94-112.

Kaztman, Rubén (1984): “Las transformaciones sectoriales del empleo en América Latina”, Revista de la CEPAL, No 24, Santiago, pp. 83-102.

Kessler, Gabriel y Vicente Espinoza (2003): “Movilidad social y trayectorias ocupacionales en Argentina: rupturas y algunas paradojas en el caso de Buenos Aires", CEPAL, División de Desarrollo Social, Serie Políticas Sociales, № 66, Santiago.

Lazzarato, Maurizio (2001): Trabajo inmaterial, formas de vida y producción de subjetividad, DP\&AA, Río de Janeiro.

Loureiro, María (1997): "Tecnocracia y política en el Brasil de hoy", Nueva Sociedad, No 152, Noviembre-diciembre, pp.1-17.

León, Arturo y Javier Martínez (2004): “La estratificación social chilena hacia fines del siglo XX”, CEPAL, División de Desarrollo Social, Serie Políticas Sociales, № 52, Santiago.

Méndez, María Luisa y Modesto Gayo (2005): “El perfil de un debate: movilidad social y meritocracia. Contribución al estudio de las sociedades latinoamericanas", en Raúl Atria, Rolando Franco y Arturo León (2007): Estratificación y movilidad social en América Latina. Transformaciones estructurales de un cuarto de siglo, LOM Ediciones, CEPAL, Santiago.

Medina Echeverría, José (1967): Aspectos sociales del desarrollo económico, CEPAL, Santiago.

(1973): Consideraciones sociológicas del desarrollo económico, Hachette, Buenos Aires.

Mora y Araujo, Manuel (2001): "Evidencias y conjeturas acerca de la estratificación actual de Argentina", CEPAL, División de Desarrollo Social, Serie Políticas Sociales, № 51, Santiago.

Moulián, Tomás (1997): Chile actual: anatomía de un mito, LOM Ediciones, Santiago.

O’Donnell, Guillermo (1975): Reflexiones sobre las tendencias de cambio del Estado burocrático autoritario, CEDES, Buenos Aires.

Pinto, Aníbal (1984): "Metropolización y tercerización. Malformaciones estructurales en el desarrollo latinoamericano", Revista de la CEPAL, № 1, Santiago, pp. 17-38.

PNUD (2008): Desarrollo humano en Chile rural, PNUD, Santiago.

Portes, Alejandro y Kelly Hoffman (2003: "Las estructuras de clase en América Latina: composición y cambios durante la época neoliberal", CEPAL, División de Desarrollo Social, Serie Políticas Sociales, № 68, Santiago. 
Sayer, Andrew y Richard Walter (1992): La nueva economía social, Ministerio del Trabajo y Seguridad Social, Madrid.

Solari, Aldo, Rolando Franco y Joel Jutckowitz (1976): Teoría, acción social y desarrollo en América Latina, Siglo XXI, México.

Ruiz, Carlos (2009): Documento de trabajo del módulo ocupación de la Encuesta de Estructura Social en la Región Metropolitana CIES 2009, Centro de Investigación de Estructura Social (CIES), Universidad de Chile, Santiago.

(2006): "América Latina y la excepcionalidad chilena, ¿Asincronías temporales o destinos divergentes?", en Rodrigo Baño (edit.): Chile en América Latina. Integración o desintegración regional en el siglo XXI. Homenaje a Enzo Faletto, Facultad de Ciencias Sociales, Universidad de Chile, Santiago, pp. 87-121.

Tokman, Víctor (1979): Dinámica de mercado de trabajo urbano: el sector informal urbano en América Latina, Colegio de México, México.

(2007): "Informalidad, inseguridad y cohesión social en América Latina", CEPAL, División de Desarrollo Social, Serie Políticas Sociales, № 130, Santiago.

Touraine, Alain (1989): América Latina, política y sociedad, Espasa-CALPE, Madrid.

OIT (2002): Actas conferencia internacional del trabajo, OIT.

Weller, Jurgen (2001): “Procesos de exclusión e inclusión laboral: la expansión del empleo en el sector terciario", CEPAL, División de Desarrollo Económico, Serie Macroeconomía del Desarrollo, $N^{\circ}$ 6, Santiago. 